Trauma Berufskrankh 2010 • 12 [Suppl 4]:421-424 DOI 10.1007/s10039-010-1613-z

Online publiziert: 18. April 2010

๑) Springer-Verlag 2010
B. Hogan 1 . G. Altrock ${ }^{2}$. C. Dodt ${ }^{3}$

${ }^{1}$ Zentrale Notaufnahme, Asklepios Klinik Hamburg Altona

${ }^{2}$ Zentrale Notaufnahme, Klinikum Offenbach GmbH

${ }^{3}$ Präklinik, Klinikum Bogenhausen, München

\title{
Akzeptanzprobleme hinsichtlich des Facharztes für Notfallmedizin
}

\section{Sicht der DGINA (Deutsche Gesellschaft für Interdisziplinäre Notfallaufnahme) e.V.}

\section{Lage der Notfallmedizin in Deutschland - anstehender Paradigmenwechsel}

Auf dem Gebiet der Notfallmedizin in Deutschland findet derzeit ein Wandel statt. Die Zahl der Notfallpatienten in Krankenhäusern steigt kontinuierlich und in vielen Regionen dramatisch, bedingt durch den demografischen Wandel und die Umstrukturierungen der kassenärztlichen Notfallversorgung.

Unter dem Titel „Zentral und interdisziplinär" stellte eine Gruppe von Experten der Fachgesellschaften (Deutsche Gesellschaft für Innere Medizin, Deutsche Gesellschaft für Chirurgie) im Deutschen Ärzteblatt vom 19.02.2010 ihren „Konsensus“ über „die inhaltliche Ausrichtung einer zentralen Notaufnahme" zur Diskussion, und bezog damit zu dem historischen Wandel in der Notfallmedizin Stellung [4]. Auf diese Veröffentlichung hin verfasste die Deutsche Gesellschaft für Interdisziplinäre Notfallaufnahme (DGINA e.V.) eine Stellungnahme [3], die die inhaltliche Grundlage des aktuellen Beitrags darstellt.

An frühere Stellungnahmen der Deutschen Gesellschaft für Innere Medizin und der Deutschen Gesellschaft für Chirurgie (November 2006, [2]) anknüpfend wurde in dem Konsensuspapier [4] erstmals die Realität interdisziplinärer Notaufnahmen und Aufnahmestationen akzeptiert, zentraler Anlaufpunkt für alle Notfallpa- tienten zu sein. Eine fachlich-räumliche Trennung wird nicht mehr gefordert, sondern eine organisatorische Zusammenfassung anerkannt. Auf diesem Wege wird nachträglich autorisiert, was die normative Kraft des Faktischen trotz der früheren Widerstände bereits an vielen Orten zur Umsetzung gebracht hat. Das Konsensuspapier wurde unter bewusster Auslassung der DGINA e.V. als Fachgesellschaft der in der Notaufnahme Tätigen erstellt. Dieser Umstand gibt den Autoren die Möglichkeit, als Vertreter der DGINA zu den fachlichen Inhalten des Konsensuspapiers unbefangen Stellung zu nehmen.

Die DGINA e.V. wurde im Bewusstsein der besonderen Verantwortung für die Weiterentwicklung einer professionellen Notfallmedizin auch in deutschen Notaufnahmen, die internationalen Maßstäben genügt, als fachliches Forum für in der Notaufnahme arbeitende Ärzte/-innen und Pflegekräfte 2005 gegründet. Seit dieser Zeit hat sich der kleine Gründungskreis von 9 Kliniken ebenso erweitert wie die Anzahl an eigenständigen zentralen Notaufnahmen gestiegen ist. Gegenwärtig ist die DGINA das Sprachrohr von annähernd 100 zentralen Notaufnahmen in Deutschland.

\section{Effiziente Notfallversorgung durch zentrale Notaufnahmen}

Die herkömmlichen „Aufnahme-Ambulanzen“ des Krankenhauses mit ihren an der Einweisungsdiagnose orientierten Prozessen stoßen an die Grenzen ihrer Möglichkeiten. Nur als „Notfallzentrum“, d. h. als kompetenter Ansprechpartner für alle „ungeplanten“ Patientenkontakte des Klinikums in der Organisationsform der Zentralen Notaufnahme (ZNA), werden sie den Ansprüchen des Gesetzgebers und der Gesellschaft gerecht. Um ein medizinisch optimales Behandlungsergebnis zu erreichen und den ökonomischen Gegebenheiten knapper Ressourcen gerecht zu werden, müssen diese Patienten sehr effizient versorgt werden. Oft werden 50$60 \%$ der stationären Patienten eines Krankenhauses über die Notaufnahme aufgenommen, hier findet innerhalb kurzer Zeit eine initiale Diagnostik statt, und es werden die Weichen für eine erfolgreiche Therapie gestellt. Somit stehen die ZNA und die dort arbeitenden Berufsgruppen in besonderer Verantwortung. Ihre Leistung ist immer ein spezifisches Qualitätsmerkmal eines Krankenhauses, das auch von der Öffentlichkeit besonders wahrgenommen wird.

Verbunden mit der Einrichtung zentraler Räumlichkeiten für die Notfallversorgung stellt sich die Frage, ob und wie die notwendige Interdisziplinarität effektiv organisiert werden kann und wer in dieser Struktur die medizinische Verantwortung $\mathrm{zu}$ übernehmen hat. In vielen Kliniken stellte sich heraus, dass die räumliche Gemeinsamkeit alleine nicht genügt, einen Interdisziplinarität zwingend erfor- 
dernden Behandlungsablauf reibungsfrei zu gestalten. Dies trifft insbesondere auf die Großkliniken zu, in denen viele Fachdisziplinen an der Notfallversorgung beteiligt sind.

\section{Primäre Fachspezifität - optimale Notfallversorgung?}

Angelsächsische Modelle des „emergency department" konnten überzeugend darlegen, wie wichtig eine straff organisierte Notfallversorgung für das Überleben der Patienten ist. Als Konsens in allen europäischen Notfallfachgesellschaften gilt, dass es nicht Aufgabe von in der Notaufnahme beschäftigten Ärzten ist, Patienten von der ihnen zustehenden fachspezifischen Behandlung fernzuhalten, sondern diese in gebotener Geschwindigkeit zu ermöglichen. In dieser Beurteilung stimmen Gries et al. [4] in ihrem Konsensuspapier mit dem Vorstand der DGINA überein. Dazu ist es erforderlich, eine diagnostische Klärung zu erreichen und bei der zunehmenden Zahl multimorbider Patienten abzuwägen, welches Gesundheitsproblem primär bzw. dringlich zu behandeln ist und welche begleitenden Gesundheitseinschränkungen besondere Risiken beinhalten.

Die Forderung, dass ein Patient fachbezogen und nach Facharztstandard durch die jeweilige Fachabteilung in der Notaufnahme behandelt werden soll, ist mit der Wirklichkeit des Krankenhausalltags nicht vereinbar. Einerseits werden viele Notaufnahmen außerhalb der Dienstzeiten der kassenärztlichen Notfallversorgung als Anlaufpraxen genutzt, in denen eine allgemeinärztliche Qualifikation erforderlich ist. Bei der geforderten alleinigen Präsenz von Teilgebietsfachärzten finden aufgrund der spezialisierten Weiterbildungsinhalte der Fachabteilungen bis zu 30\% der Patienten in der ZNA keinen zuständigen Arzt. Erfahrungsgemäß ist nur ein kleinerer Teil von Patienten einer Notaufnahme prima vista mit einer klaren fachspezifischen Diagnose zu versehen und unmittelbar einem Spezialgebiet zuzuordnen. Auch aus ökonomischen Erwägungen ist die Vorhaltung von Fachärzten aller versorgungspflichtigen Fachabteilungen eines Klinikums in der ZNA nicht sinnvoll.
Bei allen mit einem oder mehreren Leitsymptomen vorstelligen Notfallpatienten in der ZNA ist es Aufgabe des Notfallmediziners, neben der wahrscheinlichsten Diagnose alternative, gefährliche Differenzialdiagnosen rasch herauszufiltern und zeitgerecht der richtigen fachspezifischen Behandlung zuzuführen. Der Ansatz der gleichzeitigen anstatt einer sequenziellen Diagnostik erfordert primär eine breite berufliche Ausbildung und Erfahrung sowie fach- und organübergreifendes Denken.

Bedrohliche Fehler in einer Notaufnahme entstehen besonders dann, wenn frühzeitig ein Symptom fälschlich auf die häufigste fachspezifische Differenzialdiagnose zurückgeführt wird, ohne alternative risikobehaftete Diagnosen anderer Fachgebiete erwogen zu haben. Daher ist es zwingend notwendig, Ärzte/-innen in der Notaufnahme besonders auf die Kardinaltugenden einer "generalistischen“ Akutdiagnostik und der Risikostratifizierung von Erkrankungen hin auszurichten. Nur so kann das Ziel erreicht werden, zeitkritische und lebensgefährliche Krankheitsbilder auszuschließen bzw. zu verifizieren, ohne dabei die parallel notwendige Initialtherapie zur primären Stabilisierung zu vernachlässigen.

Die Spezialisten der in den Krankenhäusern vertretenen Fachabteilungen sind in der Regel außerhalb der Notaufnahme mit Aufgaben beschäftigt, die ihrer Spezialisierung innerhalb der stationären $\mathrm{Pa}-$ tientenversorgung dringender bedürfen, sodass eine unverzügliche Vorstellung ohne eindeutig zugeordnete Diagnose illusorisch ist. Es ist im Gegenteil eine der Aufgaben der Notaufnahme, Patientenströme so zu lenken, dass nur der Patient hochspezifische Leistungen erhält, der diese aufgrund der Art und Schwere der Diagnose auch benötigt. Es ist weder ökonomisch sinnvoll noch medizinisch-fachlich geboten, für jede in der DRG-Betrachtung (DRG: „diagnosis related groups") letztendlich fachspezifische Diagnose die jeweiligen Fachkollegen zu Abklärung der Frage hinzuzuziehen, ob eine stationäre Aufnahme notwendig ist oder nicht. Vielmehr ist von kompetenten Notfallmedizinern in einer Notaufnahme zu erwarten, dass sie die Indikation zu einer stationären Aufnahme stellen können und den
Patienten dann unverzüglich und korrekt den Fachabteilungen zuführen.

Auf keinen Fall kann eine effiziente Notfallmedizin sich darauf beschränken, den Akutpatienten an den jeweils am besten geeigneten Spezialisten zu vermitteln, statt verantwortlich in den Behandlungsprozess bis zu Klärung der Diagnose und Einleitung einer optimalen Therapie einzugreifen. Ein Notfallmediziner mit ausschließlich organisatorischem Mandat, wie von den Fachgesellschaften gewünscht, der keine breite medizinische Kompetenz für die in der Notaufnahme zu behandelnden Notfälle hat bzw. ausüben darf, ist ein „Papiertiger“, der sich zudem haftungsrechtlich in einer ausgesprochen unsicheren Beweislage befindet [5].

\section{Professionalisierte „innerklinische“ Notfallmedizin in Deutschland}

Für den Vorstand der DGINA steht fest, dass eine profunde Ausbildung in der Notfallmedizin weit über die präklinische Notfallmedizin herausreicht, indem sie relevante notfallmedizinische Kenntnisse der "großen“ Fachdisziplinen Chirurgie, Innere Medizin, Neurologie und Unfallchirurgie einbezieht und zusammenfasst. Fächer wie Pädiatrie, Frauenheilkunde, Urologie und Psychiatrie werden in Deutschland Sondergebiete bleiben, deren Notfallorganisation von der spezifischen lokalen Versorgungssituation abhängt. Allerdings würde eine professionalisierte Notfallmedizin nach internationalem Vorbild bei der Versorgung von Patienten aus diesen Fachgebieten in Krankenhäusern ohne die entsprechenden Fachabteilungen ebenfalls zu einer verbesserten Grundversorgung beitragen.

Unsere empirisch gut begründete Position konterkariert die in dem Statement der Fachgesellschaften im Ärzteblatt dargestellte Meinung der Sprecher dieser Fachgesellschaften [4]. So sei die heutige fachliche Qualifikation in Notfallmedizin in Deutschland ausreichend, weil

„Die fachspezifische Notfallversorgung fester Bestandteil einer jeden Facharztweiterbildung und fachspezifischer Fortbildungsprogramme" [4]

sei. Diese Aussage lässt sich anhand der aktuellen Weiterbildungsordnungen und 
-richtlinien nicht belegen und ist faktisch falsch. Lediglich die chirurgischen Fächer fordern in ihrem „common trunk“ zwingend ein halbes Jahr vollzeitige Weiterbildung in der Notaufnahme. Für die Erlangung eines internistischen Facharztes ist eine Ausbildung im Bereich der Notaufnahme anrechenbar, aber nur optional erforderlich. Die Weiterbildungsinhalte der Anästhesiologie sehen nicht einmal vor, dass die erforderlichen Kenntnisse der Diagnostik und Therapie notfallmedizinisch relevanter Erkrankungen in den jeweils fachlich zuständigen Bereichen der Krankenversorgung erlernt werden müssen.

Der 8o-stündige „Fachkunde“-Kurs, der zur Erlangung der Zusatzweiterbildung Notfallmedizin für den präklinischen Bereich vorausgesetzt wird, wurde in früheren Expertenstatements als „,common trunk" der klinischen Notfallmedizin in Erwägung gezogen. Er bildet die Anforderungen einer hochkompetenten innerklinischen Notfallmedizin an den Nochnicht-Facharzt in keiner Weise ab.

Die Weiterbildungsordnungen für die meisten am Krankenhaus vertretenen Fachdisziplinen sind diagnoseorientiert und auf Organsysteme bzw. Körperregionen fokussiert. Sie setzen die symptombezogene Notfallversorgung voraus, geben aber keinerlei Anleitung hierfür. Auch wenn alle diagnostisch und therapeutisch tätigen medizinischen Fachdisziplinen notfallmedizinische Kenntnisse in den sie betreffenden Krankheitsbildern wissenschaftlich und praktisch sicherstellen, verfügt keine für sich allein über die notwendige Breite der medizinischen Kenntnisse, die der Alltag in einer Notaufnahme in optimaler Weise erforderlich macht.

Zusätzlich besteht in Krankenhäusern der Grund- und Regelversorgung immer wieder die Notwendigkeit, auch Notfälle aus nicht am Haus vertretenen Fachdisziplinen medizinisch fachgerecht erstzuversorgen. Die hierfür benötigte Kompetenz wird derzeit in Deutschland in keiner Weise strukturiert weitergebildet. Weiterbildungsinhalte, die die sehr spezifischen Anforderungen an Notaufnahmen wie das Schnittstellenmanagement, die migrantenspezifische Medizin, den Massenanfall von Verletzten, die Grippepandemie usw. abbilden, fehlen in der Musterweiterbildungsordnung komplett.

Trauma Berufskrankh 2010 - 12 [Suppl 4]:421-424 DOI 10.1007/s10039-010-1613-z

C) Springer-Verlag 2010

B. Hogan · G. Altrock · C. Dodt

Akzeptanzprobleme hinsichtlich des Facharztes

für Notfallmedizin. Sicht der DGINA (Deutsche Gesellschaft für Interdisziplinäre Notfallaufnahme) e.V.

\section{Zusammenfassung}

Die Zahl der Notfallpatienten in Krankenhäusern steigt kontinuierlich, was zum einen auf den demografischen Wandel, zum anderen auf Umstrukturierungen der kassenärztlichen Notfallversorgung zurückzuführen ist. Im Februar 2010 wurde von Experten verschiedener Fachgesellschaften ein Konsens veröffentlicht, laut welchem interdisziplinäre Notaufnahmen und Aufnahmestationen zentraler Anlaufpunkt für alle Notfallpatienten darstellen und demzufolge eine organisatorische Zusammenfassung erfordern. Hierzu wird im vorliegenden Beitrag von Seiten der nicht an der Erstellung dieses Konsensus beteiligten DGINA e.V., der Deutschen Gesellschaft für Interdisziplinäre Notfallauf- nahme als Fachgesellschaft der in der Notaufnahme Tätigen, Stellung genommen. Es wird evident, dass die Professionalisierung der Notfallmedizin in Deutschland überfällig ist und baldmöglichst realisiert werden sollte. Um dieses zu erreichen, sollte das in der UEMS (Union Européenne des Médicins Spécialistes) konsentierte EuSEM (European Society of Emergency) Core Curriculum auch in Deutschland umgesetzt werden.

\section{Schlüsselwörter}

Interdisziplinäre Notaufnahme · Deutsche Gesellschaft für Interdisziplinäre Notfallaufnahme · Curriculum Notfallmedizin · DGINA . EuSEM Core Curriculum

\section{Acceptance problems regarding the specialist for emergency medicine. The perspective of the German Society of Interdisciplinary Emergency Departments (DGINA)}

\section{Abstract}

The number of emergency patients in hospitals is rising continuously as a result of demographic changes on the one hand and the restructuring of statutory health service emergency treatment on the other. In February 2010 a group of experts from a number of specialist medical associations published a consensus according to which interdisciplinary emergency units and emergency departments represent the central starting point for all emergency patients, and therefore require standardized organisation. The current article presents the view of the German Society of Interdisciplinary Emergency Departments (DGINA e.V.) - which was not involved in the drafting of the consensus - in its capacity as a specialist medical association active in the field of emergency care. It is clear that the professionalization of emergency medicine in Germany is overdue and should be made effective as soon as possible. In order to achieve this, the contents of the EuSEM (European Society of Emergency) core curriculum, formulated in consensus with UEMS (Union Européenne des Médicins Spécialistes), must also be put into practice in Germany.

\section{Keywords}

Interdisciplinary emergency department . German Society of Interdisciplinary Emergency Departments - Emergency medicine curriculum · DGINA · EuSEM Core Curriculum 


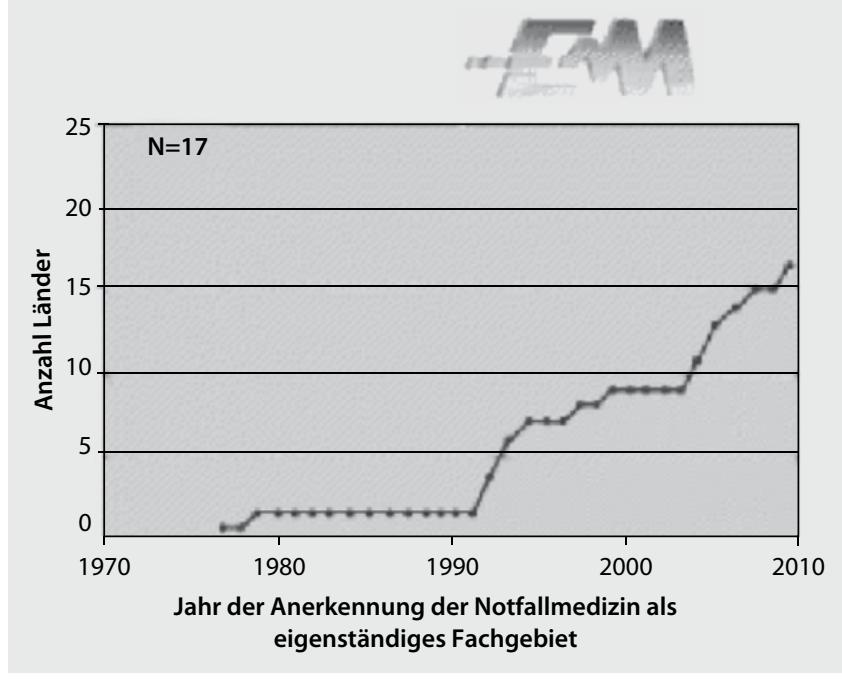

Fazit

Mit der von der DGINA vorgeschlagenen Priorisierung der Maßnahmen wird sich ein guter Weg finden lassen, die gesundheitspolitischen Potenziale der neuen Struktur Zentrale Notaufnahme zu fördern. Damit scheint auch die Problematik unzureichend genutzter Leistungsreserven in der Notfallversorgung der Krankenhäuser lösbar. Wird die Notwendigkeit der operativen und strategischen Exzellenz in dem lebensentscheidenden Bereich eines Krankenhauses betont, wird eine verstärkte Orientierung auf die Potenziale, die Prozesse und die Ergebnisse einer solchen Einrichtung möglich werden.

Aus Sicht der DGINA e.V. sollten alle beteiligten Fachdisziplinen unter der inhaltlichen Vorgabe des EuSEM-Curriculums einen tragfähigen und zukunftsweisenden Weg erarbeiten, um die überfällige Professionalisierung der Notfallmedizin in Deutschland zu realisieren.

\section{Korrespondenzadresse}

\section{Dr. B. Hogan}

Zentrale Notaufnahme,

Asklepios Klinik Hamburg Altona,

Paul-Ehrlich-Straße 1, 22763 Hamburg

b.hogan@asklepios.com

Interessenkonflikt. Der korrespondierende Autor gibt an, dass kein Interessenkonflikt besteht.

\section{Literatur}

1. BGH (2009) Urteile des BGH von 1981 , VI ZR 69/80 und VI ZR 386/96. Bundesgerichtshof, Karlsruhe

2. Deutsche Gesellschaft für Chirurgie, Deutsche Gesellschaft für Innere Medizin (2006) Zur Problematik Zentraler Notaufnahmen. Gemeinsame Stellungnahme der Deutschen Gesellschaft für Chirurgie und der Deutschen Gesellschaft für Innere Medizin, November 2006. DGIM, Wiesbaden, www. dgim.de/stellungPosMitt/pub_stellungnahmen_ pospapier.html

3. DGINA (2010) Notfallmedizin in Deutschland - Ringen um Besitzstandswahrung. Stellungnahme der DGINA. Dtsch Ärztebl im Druck

4. Gries FA, Seekamp A, Welte Tet al (2010) Zentral und interdisziplinär. Dtsch Arztebl 107(7):A268269

5. Killinger $E$ (2009) Die Besonderheiten der Arzthaftung im medizinischen Notfall. Dissertation, Juristische Fakultät, Universität Regensburg. MedR XIII:1304

6. Schlechtriemen T, Dirks B, Lackner CK et al (2005) Die „interdisziplinäre Notaufnahme“ im Zentrum zukünftiger Notfallmedizin. 10. Leinsweiler Gespräch der agswn e.V. in Verbindung mit INM, IfN und BAND. Notfall Rettungsmed 8:502-511 